\title{
Muséologies
}

Les cahiers d'études supérieures

\section{Rôle social des musées : une autre « nouvelle " muséologie}

\section{Mathieu Dormaels}

Volume 2, numéro 2, avril 2008

URI : https://id.erudit.org/iderudit/1033593ar

DOI : https://doi.org/10.7202/1033593ar

Aller au sommaire du numéro

Éditeur(s)

Association Québécoise de Promotion des Recherches Étudiantes en Muséologie (AQPREM)

ISSN

1718-5181 (imprimé) 1929-7815 (numérique)

Découvrir la revue

Citer ce compte rendu

Dormaels, M. (2008). Compte rendu de [Rôle social des musées : une autre « nouvelle » muséologie]. Muséologies, 2(2), 118-123.

https://doi.org/10.7202/1033593ar d'utilisation que vous pouvez consulter en ligne.

https://apropos.erudit.org/fr/usagers/politique-dutilisation/ 
Billet deux

\section{Rôle social des musées : une autre "nouvelle" muséologie}

[Par Mathieu Dormaels, doctorant au programme international conjoint en muséologie, médiation et patrimoine à l'Université du Québec à Montréal (Canada) et l'Université d'Avignon et des Pays de Vaucluse (France). ] 
Rôle social des musées : une autre

" nouvelle " muséologie

Depuis les années 1970, il s'est développé une nouvelle façon de penser les musées et leur relation avec leurs publics. À la suite de George-Henri Rivière, d'André Desvallées ou de Duncan Cameron ${ }^{[1]}$ sont apparues des expressions qui ont depuis fait leur chemin vers le sens commun qu'on leur connaît, et que l'on retrouve fréquemment dans les discours des institutions muséales et dans les objectifs des politiques culturelles. Ainsi parle-t-on, à tort ou à raison, de muséologie sociale ou participative, du " musée-forum " ${ }^{[2]}$ ou encore du rôle social des musées ${ }^{131}$. Ces questions sont certainement riches de réflexions et fécondes pour les musées aujourd'hui et il n'est pas de notre propos d'en discuter l'évidente pertinence, mais encore faut-il se donner les moyens d'y répondre avec l'«objectivité » qu'elles méritent.

En déplaçant le centre d'intérêt du musée vers le visiteur, on a créé une bipolarité entre, d'une part, la recherche et la conservation de la collection et, d'autre part, la "quête» d'un public diversifié qui vient apprendre et se divertir au musée. On peut considérer cette situation comme une tension, source d'une contradiction qui pousse la question du développement des musées vers le paradoxe ${ }^{[4]}$,
[1]

Voir WEISS, Hélène et Jean-

François LEROUX-DHUYS (dir.).

La Muséologie selon

Georges Henri Rivière,

Cours de muséologie,

textes et témoignages.

Paris : Dunod, 1989, 402 p.

\section{[2]}

Expression de Duncan

Cameron (1971),

en opposition au musée

"temple du beau", reprise dans

le programme du colloque

"Le rôle social des musées *

organisé par les étudiants de

muséologie, le 4 février 2008,

au Centre d'Histoire

de Montréal.
[3]

Thème du colloque

"Le rôle social des musées".

[4]

Voir LE MAREC, Joëlle.

"Les musées en devenir?

Une interrogation paradoxale».

In. SHIELE, Bernard (dir.).

Patrimoines et identités.

Québec : Musée de

la civilisation, Éditions

Multimondes, 2002, $251 \mathrm{p}$. 
ou comme un équilibre nécessaire qui garantit le bon fonctionnement du musée comme

institution permanente, sans but lucratif, au service de la société et de son développement, ouverte au public et qui fait des recherches concernant les témoins matériels de l'homme et de son environnement, acquiert ceux-là, les conserve, les communique et notamment les expose à des fins d'études, d'éducation et de délectation ${ }^{[5]}$.

Dans tous les cas, cette évolution introduit l'individu dans les préoccupations du musée, mais pas forcément dans le musée. Et, après le déclin des écomusées, c'est un autre coup porté par la «réalité quotidienne» de la majorité des institutions à cette muséologie puisque, à partir de ce nouvel intérêt, ont surgi d'autres outils tels que la promotion publicitaire, le marketing ou d'autres stratégies de gestion. Est-ce une mauvaise chose? Ce débat n'est certainement pas clos, et il n'a pas sa place ici, mais il reste que chacun est conscient qu'aujourd'hui ce n'est plus vraiment un choix que les musées peuvent se permettre. Cependant, on voit bien que le pôle de la "conservation » qui conçoit les expositions, tout comme le pôle du "service des publics", cherchent à connaître ce visiteur. On observe donc des études sur son comportement et son attitude (stratégies de lecture, de visite, etc.), d'un côté, et des statistiques sur son origine géographique, son âge ou sa profession, de l'autre. Pourtant, chacun se doit de travailler avec un "visiteur modèle", voire "virtuel", sorte de chimère ou de légende, indéfinissable et inobservable. Il devient bien compliqué dans ce cas de bâtir une "muséologie participative " qui soit autre chose que des actions ponctuelles développées au cas par cas.

Mais ne reste-il pas au musée sa collection? On s'en doute, la réalité n'est pas si simple. Car, dans un mouvement conjoint à celui de l'élargissement de la notion de patrimoine, la définition même de la collection, élément consubstantiel au musée occidental, évolue bien rapidement. Sans entrer dans les détails de cette évolution, on voit que l'émergence de l'immatérialité peut poser un certain nombre de

[5]

Définition de l'ICOM

(International Council Of Museums), article 2-1 de ses statuts, amendés lors de sa $20^{\circ}$ Assemblée générale (Barcelone, Espagne, 6 juillet 2001). 
" défis » en termes de protection et de conservation du patrimoine. Mais elle est aussi la source de nouvelles possibilités de développement et, au patrimoine intangible, commencent à faire écho les musées virtuels. Que ce soit sur les plans de la recherche et des techniques ${ }^{[6]}$, ou dans la création même de ces musées ${ }^{[7]}$ on voit se développer de nouveaux outils, de nouveaux "lieux" de conservation, de diffusion et même d'expression. Cela influence notre perception des musées en général, rendant encore plus difficile leur tâche d'attirer le public et déclanchant l'incompréhension devant l'absence d'un grand nombre de musées sur le Web. Le patrimoine se dématérialise et le musée se virtualise... Mais où sont ceux qui font le musée? Parce que même si chacun peut laisser son histoire ou sa mémoire, en ligne ou ailleurs, on ne fait là que constituer une " collection" et il est certain qu'il faudra toujours quelqu'un pour la préserver, faire des recherches et diffuser ces connaissances.
Dès lors, quelle est la place, ou quel pourrait être, dans le futur, le rôle des muséologues au sein des musées?

Avant de tenter de répondre à cette question, il convient de s'attarder un peu plus sur les personnes qui travaillent dans la grande majorité des musées, ceux de petite taille. En effet, comment continuer à travailler avec les notions, pourtant récentes, de la nouvelle muséologie quand le visiteur se fait virtuel, la collection en partie intangible et que le musée lui-même est en pleine transformation? Les professionnels des musées ont tendance à s'attacher aux principes et aux techniques muséologiques qui sont perçus comme un socle de stabilité et de constance dans ce contexte. Or, on voit bien que les actions qu'entreprennent les musées, sur la base de ces principes de muséologie sociale et participative, sont de leur initiative et pas de celle des communautés.

\section{[6]}

Voir le communiqué de presse du 28 mai 2007 de l'Université Laval (Québec), annonçant la création du Laboratoire de muséologie et d'ingénierie de la culture (LAMIC), <http:// relationsmedias.ulaval.ca/ comm/2007/mai/nouveaulaboratoire-museologieexperimentale-111.htm $D$ (consulté le 8 février 2008).

\section{[7]}

Voir par exemple le Musée virtuel de la personne, <http://www.museudapessoa. net/MuseuVirtual/home/ resources/homesPublicadas/ MVHM_4.html>. 
Quelle est leur légitimité, en termes "sociaux" et "participatifs", à mener de tels projets ? Il va de soi qu'on ne saurait remettre en cause l'acquisition, la conservation et la mise en exposition d'objets - matériels et immatériels d'une communauté ou d'un groupe, mais il est notable qu'on le fasse dans cette perspective sociale, sans se demander s'il est vraiment pertinent d'utiliser ce modèle. C'est le type de situation dans laquelle on essaie de faire un musée, dont la collection et l'exposition seraient représentatives d'une communauté particulière, sans se demander si le fait même de faire un musée est pertinent dans cette culture. Ainsi les évolutions récentes de la notion de patrimoine, des possibilités de la technologie et de l'augmentation de l'accessibilité des musées, ont pris de court la muséologie et son enseignement, parce que, enfin, on se rend bien compte des dérives possibles liées à ces changements. Les musées, rattrapés par les chiffres et la "démocratisation" de la culture - principe pourtant louable en soi -, se retrouvent coincés dans l'obligation d'être ouverts à tous et de justifier leur grande accessibilité aux organismes, privés ou publics, qui les financent et ce, par une fréquentation diversifiée et toujours en hausse. Cependant, à vouloir attirer tout le monde, tout le temps, et par les mêmes techniques, on en arrive à se demander dans quelle mesure les musées ne proposent-ils pas la "même chose". S'il y a un principe de marketing que ces musées pourraient appliquer, c'est de définir la spécificité de leur offre et leur « avantage concurrentiel ${ }^{\left[{ }^{[3]}\right.}$.

Que peut donc être ce " rôle social du musée " ? L'institution muséale est sans conteste implantée dans une communauté et elle a des responsabilités envers cette société. Une de ces responsabilités est sans doute de favoriser l'accès pour tous à ses installations. Mais chaque lieu est différent, chaque musée 
particulier, au-delà des typologies, en raison de son histoire, de son contenu et de son environnement physique et social. C'est donc un " contrat " particulier qui est passé entre le musée et la société, et c'est ce qui définit sa «mission». C'est aussi ce qui doit être la base de son fonctionnement, lélément de stabilité sur lequel se construisent son projet et ses objectifs. Autrement dit, «l'énoncé de la mission attire notre attention vers les raisons les plus permanentes et les plus profondes de l'existence du musée. C'est le fondement de n'importe quelle ligne d'action ou développement postérieur ${ }^{[09}$." Car, même du point de vue des gestionnaires, «identifier la mission correcte peut prendre du temps, mais c'est essentiel pour diriger l'institution à long terme car la mission est le noyau autour duquel doivent se développer les politiques de l'institution " ${ }^{(10)}$. Ce que peuvent faire les muséologues au sein des musées - et concrètement de l'intérieur ou de l'extérieur -, c'est participer à la définition de la mission et des objectifs, ou à son actualisation le cas échéant, et développer des outils conceptuels et pratiques qui aident ces institutions à fonctionner. Grâce à cela, on pourra clarifier la place de chaque musée et ses responsabilités par rapport à la société, évaluer son action de façon pertinente et efficace - c'està-dire de manière à ce que cela soit source de progrès pour l'institution - et, ainsi, on saura lui permettre de remplir pleinement son "rôle social». 